\title{
First order sea clutter cross section for bistatic shipborne HFSWR
}

\author{
Yongpeng Zhu, Yinsheng Wei*, and Peng Tong \\ School of Electronics and Information Engineering, Harbin Institute of Technology, Harbin 150001, China
}

\begin{abstract}
This paper studies the development on the first order sea clutter cross section for bistatic high frequency surface wave radar (HFSWR). Based on the received first order electric field expression, a closed-form of cross sections is derived to account for the case of receiving antenna array being mounted on the shipborne platform. The uniform linear motion and sway motion components are assumed to be responsible for the observed differences in comparison with the bistatic fixed antenna case. Correspondingly, simulations are conducted to study the sea clutter spectral characteristics for these two cases versus different system parameters and sea state conditions. It is shown numerically that the forward motion component will spread the Bragg lines severely and the influence triggered by the sway motion can be explained as the Bessel function modulation of the ordinary sea clutter spectra. The obtained results have important implications in the application of shipborne HFSWR technology to ocean remote sensing and target detection.
\end{abstract}

Keywords: radar cross section (RCS), remote sensing by radar, sea clutter, high frequency surface wave radar (HFSWR).

DOI: $10.21629 /$ JSEE.2017.04.07

\section{Introduction}

The high frequency (HF) radar has been used for target detection over-the-horizon and remote sensing of the ocean surface for many years $[1-3]$. This is due to the fact that the sea water has a good conductivity of the vertically polarized electromagnetic wave working in the HF band (3$30 \mathrm{MHz}$ ), so that it can follow the air-water interface and adhere to the curvature of the earth at a very low propagation loss. Compared with the high frequency surface wave radar (HFSWR) systems for the case of co-location of transmitters and receivers, the bistatic deployment can address the problems such as the ambiguous sea state information elimination and suppressing mutual interference between antennas for monostatic radar system $[4,5]$.

\footnotetext{
Manuscript received April 07, 2016.

*Corresponding author.

This work was supported by the National Natural Science Foundation of China (61471144).
}

However, the conventional land-based deployment is still an intrinsic limitation on the agility and maneuverability of HFSWR. Therefore, as a further development, the condition of receiving antenna array being mounted on a shipborne platform may enlarge applicable regions.

As the most dominant clutter component of HFSWR, the well-known Bragg peaks produced by the first order scattering interaction with the Bragg waves not only constitute the primary clutter background of target detection but also lay the foundation of ocean remote sensing [5,6]. However, because of the additional Doppler modulation imposed by the shipborne platform motion, it is reasonable to deduce the enhanced clutter spectral complexity. Intuitively, towards a full understanding of sea clutter characteristics, a model to explain the intrinsic modulation mechanism is needed.

Based on the semisubmersible oil platform, Lipa et al. carried out experiments to extract the wave height directional spectrum parameters and derived a method to eliminate the effects of phase-modulation contamination imposed by the platform motion [7]. Allowing for the nonideal motions, Lesturgie analysed the impact of pitch and roll motions on the detection of slow targets in shipborne HFSWR and concluded that when the ship was moving, these motions did not modify the shape of the sea clutter spectrum significantly [8]. Subsequently, Walsh et al., on the basis of received electric field scattering from the sea surface, yielded the first and second order radar ocean surface cross sections applied for an antenna mounted on a floating barge [9,10]. It was illustrated that the effect of sway motion could be regarded as an amplitude-frequency modulation of the Bragg peaks. Given the complexity of the Walsh's method, Khoury et al. considered the backscattered received signals as a combination of weighting contributions from all antenna elements so as to yield a simplified approach to simulate the sea-echo Doppler spectrum [11]. Xie et al. developed a Walsh's model on condition that the ship was keeping the uniform linear motion and 
discussed about the potential for sea state parameter measurement by shipborne HFSWR [12,13]. Further, associated with the rank analysis in [14], Xie also studied the sparsity signatures of first order sea clutter and yielded a method to estimate the high resolution space-time spectrum using a technique combining the sparse representation and multiple signal classification (MUSIC) algorithm [15]. However, their analysis only focused on the monostatic case.

Based upon the modified electric field equation, the first order sea clutter cross section is theoretically derived in Section 2 for bistatic shipborne HFSWR. In Section 3, two conditions that the ship is assumed to be in uniform linear motion and sway motion are considered, so as to yield an analytical expression to calculate the resultant cross sections. Section 4 provides illustrations and analysis of the derived cross sections for different system parameters and sea environment conditions. Eventually, a brief summary is given in Section 5.

\section{First order scatter}

\subsection{Modified first order field equation}

The background of scattered electric field equation from rough surfaces incorporating a special excitation source is given by [16]. The technique considered in this paper is referred to the Walsh's model with an HF vertical pulse diploe source, which is then developed for the case of receiving antenna array being mounted on a shipborne platform.

In the Walsh's method [16], that the rough ocean surface is good conducting, and small surface heights and small slopes are throughout imposed. After the Neumann series approximation to the electric field equation, the first order scattered electric field is

$$
\begin{gathered}
\left(E_{0 n}^{+}\right)_{1} \approx \frac{k C_{0}}{(2 \pi)^{2}} \sum_{\boldsymbol{K}}{ }_{1} P_{\boldsymbol{K}, \omega} K . \\
\int_{x_{1}} \int_{y_{1}} \cos \left(\theta_{1}-\theta_{\boldsymbol{K}}\right) \frac{F\left(\rho_{1}\right) F\left(\rho_{2}\right)}{\rho_{1} \rho_{2}} . \\
\mathrm{e}^{\mathrm{j} \rho_{1} K \cos \left(\theta_{1}-\theta_{\boldsymbol{K}}\right)} \mathrm{e}^{-\mathrm{j} k\left(\rho_{1}+\rho_{2}\right)} \mathrm{d} x_{1} \mathrm{~d} y_{1}
\end{gathered}
$$

where $C_{0}=I \Delta l k^{2} /\left(\mathrm{j} \omega \epsilon_{0}\right)$ indicates the source current $I$ with a dipole of length $\Delta l$ whose wave number is $k$ ( $k=\omega / c, c$ is the velocity of light and $\omega$ is the radian frequency), $\epsilon_{0}$ is the permittivity of free space, $F(\cdot)$ is the Sommerfeld attenuation function, $\boldsymbol{K}$ is the first order sea wave vector with the magnitude value of $K,{ }_{1} P_{K, \omega}$ is the Fourier coefficient of first order time varying surface features, $\rho_{1}$ and $\rho_{2}$ are the distances from the scattering patch to the transmitter and receiver, $\theta_{1}$ and $\theta_{\boldsymbol{K}}$ are the angles of the vector $\boldsymbol{\rho}_{1}$ and $\boldsymbol{K}$ with respect to the $x$ axis.
Next we would like to yield a modified form of (1) when we consider the propagation path variation due to ship motion displacement. We denote $\delta \rho$ as the ship motion vector, $\theta_{\rho}$ is the supplementary angle between $\boldsymbol{\rho}_{2}$ and $\delta \boldsymbol{\rho}$, and $\theta_{v}$ is the angle of incident clutter wave direction. As illustrated in Fig. 1, when the ship is moving, the geometrical propagation path variation of $\rho_{2}$ can be approximately estimated by

$$
\begin{gathered}
\rho_{2}^{\prime}=\sqrt{\rho_{2}^{2}+(\delta \rho)^{2}+2 \rho_{2} \delta \rho \cos \theta_{\rho}} \approx \\
\rho_{2} \sqrt{1+2 \frac{\widehat{\boldsymbol{\rho}}_{2} \cdot \delta \boldsymbol{\rho}}{\rho_{2}}} \approx \rho_{2}+\widehat{\boldsymbol{\rho}}_{2} \cdot \delta \boldsymbol{\rho}
\end{gathered}
$$

where $\widehat{\boldsymbol{\rho}}_{2}$ is the direction of $\rho_{2}$ with the unit magnitude. Therefore, (1) is reasonable to be recast as

$$
\begin{gathered}
\left(E_{0 n}^{+}\right)_{1} \approx \frac{k C_{0}}{(2 \pi)^{2}} \sum_{\boldsymbol{K}}{ }_{1} P_{\boldsymbol{K}, \omega} K . \\
\int_{x_{1}} \int_{y_{1}} \cos \left(\theta_{1}-\theta_{\boldsymbol{K}}\right) \frac{F\left(\rho_{1}\right) F\left(\rho_{2}\right)}{\rho_{1} \rho_{2}} . \\
\mathrm{e}^{\mathrm{j} \rho_{1} K \cos \left(\theta_{1}-\theta_{\boldsymbol{K}}\right)} \mathrm{e}^{-\mathrm{j} k\left(\rho_{1}+\rho_{2}+\widehat{\boldsymbol{\rho}}_{2} \cdot \delta \boldsymbol{\rho}\right)} \mathrm{d} x_{1} \mathrm{~d} y_{1} .
\end{gathered}
$$

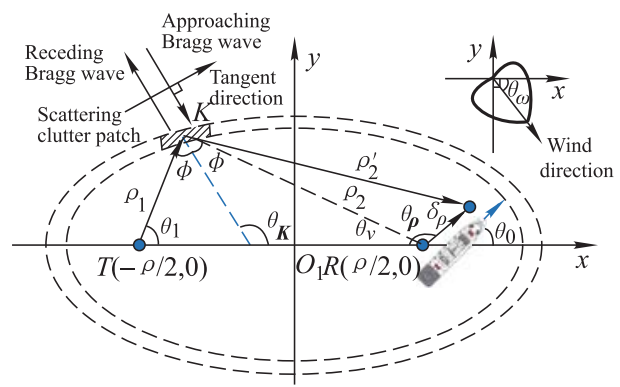

Fig. 1 Bistatic geometry propagation path variation due to the shipborne platform movement

\subsection{First order cross section derivation involving the shipborne platform motion}

According to Fig. 1, the geometry subjects to the ellipse distribution. Therefore, for the sake of convenience, we introduce the elliptic coordinate variables $\left(\mu_{0}, \delta_{0}\right)$ (a constant $\mu_{0}$ actually corresponds to a particular ellipse, $\left.\delta_{0} \in[0,2 \pi)\right)$. By doing this, $\rho_{1}$ and $\rho_{2}$ could be recast as $\rho_{1}=\rho\left(\cosh \mu_{0}+\cos \delta_{0}\right) / 2, \rho_{2}=\rho\left(\cosh \mu_{0}-\cos \delta_{0}\right) / 2$ respectively so that the modified first order scattered electric field equation can be reduced into (4) in conjunction with the notation of $\rho_{s}=\left(\rho_{1}+\rho_{2}\right) / 2=\rho \cosh \mu_{0} / 2$ and $\mathrm{d} \rho_{s}=\sqrt{\rho_{s}^{2}-(\rho / 2)^{2}} \mathrm{~d} \mu_{0}$

$$
\begin{gathered}
\left(E_{0 n}^{+}\right)_{1} \approx \frac{k C_{0}}{(2 \pi)^{3 / 2}} \sum_{\boldsymbol{K}}{ }_{1} P_{\boldsymbol{K}, \omega} \sqrt{K \cos \phi} . \\
\mathrm{e}^{\mathrm{j} \frac{\rho}{2} \cdot K} \mathrm{e}^{-\mathrm{j} \frac{\pi}{4}} \int_{\rho / 2}^{\infty} \frac{F\left(\rho_{1}, \omega\right) F\left(\rho_{2}, \omega\right)}{\sqrt{\rho_{s}\left[\rho_{s}^{2}-\left(\frac{\rho}{2}\right)^{2}\right]}} .
\end{gathered}
$$




$$
\mathrm{e}^{\mathrm{j} \rho_{s} K \cos \phi} \mathrm{e}^{-\mathrm{j} \frac{2 \rho_{s} \omega}{c}} \mathrm{e}^{\mathrm{j} \frac{\hat{\rho}_{2} \cdot \delta \rho \omega}{c}} \mathrm{~d} \rho_{s} .
$$

$\phi=\arctan \left\{\sin \left(\theta_{v}\right) /\left[1 / e_{0}-\cos \left(\theta_{v}\right)\right]\right\}$ is the half bistatic angle, where $e_{0}$ is the ellipse eccentricity, $\rho$ is the distance vector with the direction pointing from the transmitter to the receiver, whose magnitude value of baseline distance is $\rho$.

Afterwards, the time domain analysis is conducted by taking the inverse temporal Fourier transform $F_{t}^{-1}(\cdot)$ of (4). As $\omega$ is the transformed time variable, it is reasonable to deduce that $F_{t}^{-1}\left\{k C_{0}\right\}^{t}{ }^{t} F_{t}^{-1}\left\{\mathrm{e}^{-\mathrm{j} \frac{2 \rho_{s} \omega}{c}+\mathrm{j} \frac{\hat{\rho}_{2} \cdot \delta \rho \omega}{c}}\right\}$ is the one determining the final temporal field form essentially. Particularly, we consider (4) on a condition of the pulse dipole excitation source with the pulse width $\tau_{0}$ working at $\omega_{0}$ and thus we have

$$
\begin{aligned}
& F_{t}^{-1}\left\{k C_{0}\right\}^{t} F_{t}^{-1}\left\{\mathrm{e}^{-\mathrm{j} \frac{2 \rho_{s} \omega}{c}+\mathrm{j} \frac{\hat{\rho}_{2} \cdot \delta \rho \omega}{c}}\right\}= \\
& -\mathrm{j} \eta_{0} I \Delta l k_{0}^{2} \mathrm{e}^{\mathrm{j} \omega_{0}\left(t-\frac{2 \rho_{s}}{c}+\frac{\hat{\boldsymbol{\rho}}_{2} \cdot \delta \rho}{c}\right)} . \\
& {\left[h\left(t-\frac{2 \rho_{s}}{c}+\frac{\widehat{\boldsymbol{\rho}}_{2} \cdot \delta \boldsymbol{\rho}}{c}\right)-\right.} \\
& \left.h\left(t-\frac{2 \rho_{s}}{c}+\frac{\widehat{\boldsymbol{\rho}}_{2} \cdot \delta \boldsymbol{\rho}}{c}-\tau_{0}\right)\right]
\end{aligned}
$$

where $t$-star indicates the time convolution, $\eta_{0}=1 /\left(\epsilon_{0} c\right)$ is the intrinsic impedance of free space and the Heaviside function $h(\cdot)$ is

$$
\begin{gathered}
{\left[h\left(t-\frac{2 \rho_{s}}{c}+\frac{\widehat{\boldsymbol{\rho}}_{2} \cdot \delta \boldsymbol{\rho}}{c}\right)-\right.} \\
\left.h\left(t-\frac{2 \rho_{s}}{c}+\frac{\widehat{\boldsymbol{\rho}}_{2} \cdot \delta \boldsymbol{\rho}}{c}-\tau_{0}\right)\right]= \\
\left\{\begin{array}{l}
1, \quad \frac{c\left(t-\tau_{0}\right)}{2}+\frac{\widehat{\boldsymbol{\rho}}_{2} \cdot \delta \boldsymbol{\rho}}{2} \leqslant \rho_{s} \leqslant \frac{c t}{2}+\frac{\widehat{\boldsymbol{\rho}}_{2} \cdot \delta \boldsymbol{\rho}}{2} \\
0, \text { otherwise }
\end{array} .\right.
\end{gathered}
$$

Associated with (6), the limit of integration in (4) can be restricted into the interval $\left[\frac{c\left(t-\tau_{0}\right)}{2}+\frac{\widehat{\boldsymbol{\rho}}_{2} \cdot \delta \boldsymbol{\rho}}{2}, \frac{c t}{2}+\right.$ $\left.\frac{\widehat{\boldsymbol{\rho}}_{2} \cdot \delta \boldsymbol{\rho}}{2}\right]$. By invoking (4)-(6), the first order electric field expression in the time domain is yielded by

$$
\begin{gathered}
\left(E_{0 n}^{+}\right)_{1}(t) \approx \frac{\eta_{0} \Delta l I k_{0}^{2} F\left(\rho_{1}, \omega_{0}\right) F\left(\rho_{2}, \omega_{0}\right)}{\mathrm{j}(2 \pi)^{3 / 2} \sqrt{\rho_{s}\left[\rho_{s}^{2}-\left(\frac{\rho}{2}\right)^{2}\right]}} \\
\Delta \rho_{s} S a\left[\frac{\Delta \rho_{s}}{2}\left(\frac{K}{\cos \phi}-2 k_{0}\right)\right] \sqrt{K \cos \phi} \cdot \\
\sum_{\boldsymbol{K}}{ }_{1} P_{\boldsymbol{K}, \omega} \mathrm{e}^{\mathrm{j} \omega t} \mathrm{e}^{\mathrm{j}\left(\frac{\rho}{2} \cdot K+\rho_{s} \boldsymbol{K} \cos \phi+k_{0} \Delta \rho_{s}+\frac{\hat{\rho}_{2} \cdot \delta \rho}{2} \frac{K}{\cos \phi}-\frac{\pi}{4}\right)}
\end{gathered}
$$

where $S a(\cdot)$ denotes the sinc function, and $\Delta \rho_{s}$ is the scattering clutter patch size determined by the pulse width $\tau_{0}$.

The autocorrelation function is calculated by

$$
R(\tau)=\frac{A_{r}}{2 \eta_{0}}\left\langle\left(E_{0 n}^{+}\right)_{1}(t+\tau)\left(E_{0 n}^{+}\right)_{1}^{*}(t)\right\rangle .
$$

In (8), $A_{r}=\left(\lambda^{2} / 4 \pi\right) G_{r}$ is the receiving antenna area, where $G_{r}$ is the antenna gain and $\lambda$ is the radar wavelength, $\langle\cdot\rangle$ indicates the statistical average, $(\cdot)^{*}$ is the complex conjugation operation.

The Fourier transform of (8) with regard to a time shift $\tau$ gives the received power density spectrum. And our desired first order sea clutter cross section can be calculated by normalizing the power density spectrum in a per unit area sense, which is simplified as

$$
\begin{gathered}
\sigma_{11}\left(\omega_{d}\right)=2^{4} \pi k_{0}^{2} \sum_{m= \pm 1} S_{1}(m \boldsymbol{K}) \frac{K^{5 / 2} \cos \phi}{\sqrt{g}} . \\
\Delta \rho_{s} S a^{2}\left[\frac{\Delta \rho_{s}}{2}\left(\frac{K}{\cos \phi}-2 k_{0}\right)\right] \cdot \\
\int_{\tau} \mathrm{e}^{-\mathrm{j}\left(m \sqrt{g K} \tau+\omega_{d} \tau\right)}\left\langle\mathrm{e}^{\mathrm{j} \frac{K}{2 \cos \phi}\left[\widehat{\boldsymbol{\rho}}_{2} \cdot \delta \boldsymbol{\rho}(t+\tau)-\widehat{\boldsymbol{\rho}}_{2} \cdot \delta \boldsymbol{\rho}(t)\right]}\right\rangle \mathrm{d} \tau
\end{gathered}
$$

where $g$ is the gravity acceleration, $S_{1}(m \boldsymbol{K})$ is the ocean first order directional wave height spectrum determined by the product of the Pierson-Moskowitz (PM) spectrum $S_{P M}(K)$ and modified cardioid directional factor $f\left(\theta_{N}+\right.$ $\left.\psi-\theta_{\omega}\right)[3,12]$, which can be expressed as

$$
S_{1}(m \boldsymbol{K})=S_{P M}(K) f\left(\theta_{N}+\psi-\theta_{\omega}\right) .
$$

In $(10), S_{P M}(K)=0.005 \mathrm{e}^{\left[-0.74 g^{2} /\left(K^{2} U^{2}\right)\right]} / K^{4}$ and $f\left(\theta_{N}+\psi-\theta_{\omega}\right)=\frac{\xi+(1-\xi) \cos ^{4}\left[\left(\theta_{N}+\psi-\theta_{\omega}\right) / 2\right]}{2 \pi \xi+(3 \pi / 4)(1-\xi)}$, where $U$ is the wind speed, $\theta_{N}$ is the angle normal to ellipse at the clutter scattering patch, $\theta_{\omega}$ is the wind direction with respect to $x$ axis as shown in Fig. $1, \psi=$ $\left\{\begin{array}{ll}0, & m=-1 \\ \pi, & m=1\end{array}\right.$, and $\xi=0.004$ is the strength ratio of upwind returns to downwind returns.

\section{Closed-form solution for two specific scenarios}

By invoking (9), it can be analytically calculated when the ship motion is specified. In the following, two particular cases about the motion vector $\delta \rho$ are considered in order to yield a closed-form solution of sea clutter cross sections.

\subsection{Uniform linear motion}

Borne out by the experiment [2], the forward linear motion is a primary factor determining the spread spectral characteristics. Afterwards, given an ideal 
condition, we assume that the ship keeps the uniform linear motion at a constant speed $v_{0}$, i.e. $\delta \boldsymbol{\rho}=v_{0} t \widehat{\boldsymbol{\rho}}$. From $\lim _{A \rightarrow \infty} A S a^{2}(A x)=\pi \delta(x)$, the squared sampling function in (9) is reasonable to be reduced into $S a^{2}\left(\frac{\Delta \rho_{s}}{2}\left(\frac{K}{\cos \phi}-2 k_{0}\right)\right)=$ $\frac{2 \pi \cos \phi \delta\left(K-2 k_{0} \cos \phi\right)}{\Delta \rho_{s}}$. With the uniform linear motion assumption, the factored form of cross sections is given by

$$
\begin{gathered}
\sigma_{11}\left(\omega_{d}\right)=2^{5} \pi^{2} k_{0}^{2} \sum_{m= \pm 1} S_{1}(m \boldsymbol{K}) \frac{K^{5 / 2} \cos ^{2} \phi}{\sqrt{g}} . \\
\delta\left(\omega_{d}+m \sqrt{2 k_{0} g \cos \phi}-k_{0} v_{0} \cos \theta_{\rho}\right) .
\end{gathered}
$$

In (11), according to the property of the Dirac delta function $\delta(\cdot)$, the approaching and receding Bragg frequencies are given by

$$
\omega_{d}= \pm \sqrt{2 g k_{0} \cos \phi}+k_{0} v_{0} \cos \theta_{\rho} .
$$

Obviously, the finally obtained Bragg frequency is spatially modulated by the movement of the shipborne platform. That is to say, in each scattering clutter patch at a given azimuth, the forward motion will bring in an additional Doppler shift to the theoretical bistatic Bragg frequency. Therefore, as for the result received by one element, it can be regarded as the sea clutter echo superposition from omni-direction. In the Doppler domain, the sea clutter Doppler spectra spreads significantly in comparison with the case of the fixed platform condition. In addition, different from the monostatic shipborne HFSWR, the Doppler shift imposed by the ship motion is expressed as $v_{0} \cos \theta_{\rho} / \lambda$ which is the same as [17].

\subsection{Complicated case considering the non-ideal motion}

To investigate the first order sea clutter signatures under a relatively more realistic condition, the sea wave motion impact is considered. According to [11], the sway motion is modelled as

$$
\delta \boldsymbol{\rho}_{s}=a_{s} \sin \left(\omega_{s} t\right) \widehat{\boldsymbol{\rho}}_{s}
$$

where $a_{s}$ is the sway amplitude, $\omega_{s}$ indicates the dominant sea wave frequency, $\widehat{\boldsymbol{\rho}}_{s}$ is the displacement vector due to the sway motion with unit magnitude. Under this assumption, the motion vector should be recast as the vector superposition of uniform linear motion and sway motion, i.e. $\delta \boldsymbol{\rho}=a_{s} \sin \left(\omega_{s} t\right) \widehat{\boldsymbol{\rho}}_{s}+v_{0} t \widehat{\boldsymbol{\rho}}_{0}$, so that the integration item in (9) is modified as

$$
\int_{\tau} \mathrm{e}^{-\mathrm{j}\left(m \sqrt{g K} \tau+\omega_{d} \tau\right)}\left\langle\mathrm{e}^{\mathrm{j} \frac{K}{2 \cos \phi}\left[\widehat{\boldsymbol{\rho}}_{2} \cdot \delta \boldsymbol{\rho}(t+\tau)-\widehat{\boldsymbol{\rho}}_{2} \cdot \delta \boldsymbol{\rho}(t)\right]}\right\rangle \mathrm{d} \tau=
$$

$$
\begin{gathered}
\int_{\tau} \mathrm{e}^{-\mathrm{j}\left(m \sqrt{g K} \tau+\omega_{d} \tau\right)} . \\
\left\langle\mathrm{e}^{\mathrm{j} \frac{K}{2 \cos \phi}\left[v_{0} \tau \cos \theta_{\rho}+v \cos \left(\omega_{s} t+\omega_{s} \tau / 2\right)\right]}\right\rangle \mathrm{d} \tau
\end{gathered}
$$

where $v=2 a_{s} \cos \theta_{s} \sin \left(\omega_{s} \tau / 2\right), \theta_{s}$ is the angle between $\delta \rho_{s}$ and $\rho_{2}$.

Again, combining $K=2 k_{0} \cos \phi$ and $\int_{0}^{2 \pi} \mathrm{e}^{\mathrm{j} \nu \cos \vartheta} \mathrm{d} \vartheta /(2 \pi)=J_{0}(\nu),(14)$ is recast as

$$
\begin{gathered}
\int_{\tau} \mathrm{e}^{-\mathrm{j}\left(m \sqrt{g K} \tau+\omega_{d} \tau\right)} . \\
\left\langle\mathrm{e}^{\mathrm{j} \frac{K}{2 \cos \phi}\left[v_{0} \tau \cos \theta_{\rho}+v \cos \left(\omega_{s} t+\omega_{s} \tau / 2\right)\right]}\right\rangle \mathrm{d} \tau= \\
\int_{\tau} \mathrm{e}^{-\mathrm{j}\left(m \sqrt{g K} \tau+\omega_{d} \tau-k_{0} v_{0} \tau \cos \theta_{\rho}\right)} . \\
J_{0}\left[2 a_{s} k_{0} \cos \left(\theta_{s}\right) \sin \left(\omega_{s} \tau / 2\right)\right] \mathrm{d} \tau
\end{gathered}
$$

where $J_{n}(\cdot)$ is the $n$-order Bessel function. Because of the property of Bessel function $J_{0}\left[2 x \sin \left(\phi_{x} / 2\right)\right]=J_{0}^{2}(x)+$ $2 \sum_{n=1}^{\infty} J_{n}^{2}(x) \cos \left(n \phi_{x}\right)$, the cross sections involving the influence of sway motion are

$$
\begin{gathered}
\sigma_{11}\left(\omega_{d}\right)=2^{5} \pi^{2} k_{0}^{2} \sum_{m= \pm 1} S_{1}(m \boldsymbol{K}) \frac{K^{5 / 2} \cos ^{2} \phi}{\sqrt{g}} . \\
{\left[J_{0}^{2}\left(a_{s} k_{0} \cos \theta_{s}\right) .\right.} \\
\delta\left(\omega_{d}+m \sqrt{2 k_{0} g \cos \phi}-k_{0} v_{0} \cos \theta_{\rho}\right)+ \\
\sum_{n= \pm 1}^{\infty} J_{n}^{2}\left(a_{s} k_{0} \cos \theta_{s}\right) . \\
\left.\delta\left(\omega_{d}+m \sqrt{2 k_{0} g \cos \phi}-k_{0} v_{0} \cos \theta_{\rho}+n \omega_{s}\right)\right] .
\end{gathered}
$$

In (16), the sway motion effect can be explained as the Bessel function modulation of the ordinary sea clutter spectra in both Doppler and amplitude. In particular, (16) is reduced into (11) if there is no sway motion, i.e. $a_{s}=0$. From the Dirac delta function constraints, the Doppler values associated with these modulated peaks are calculated by

$$
\omega_{d}= \pm \sqrt{2 g k_{0} \cos \phi} \pm n \omega_{s}+k_{0} v_{0} \cos \theta_{\rho}
$$

According to (17), it is conceivable that each modulated Bragg peak due to the sway motion will be located symmetrically with respect to the Bragg line position calculated by (12). 


\section{Simulation and discussion}

The shipborne HFSWR system under consideration is supposed to be moving at a constant speed $v_{0}=10$ knots, working at $f_{0}=5 \mathrm{MHz}$ with a side-looking uniform linear array of 10 elements, where the element interval is half wavelength. Besides, the elementary detection and sea environment parameters are assumed as follows: the ellipse eccentricity $e_{0}=0.1$, the wind speed $U=15$ knots, the wind direction $\theta_{\omega}=0^{\circ}$ and the ship is assumed to be moving towards the positive direction of $x$ axis as shown in Fig. 1 (i.e. $\theta_{0}=0^{\circ}$, where $\theta_{0}$ is the angle between the ship motion orientation and the baseline).

\subsection{Simulated space-time distribution and spread Doppler range analysis}

By invoking (12), the space-time distributions of the approaching and receding Bragg peaks, notated as $\omega_{d}^{+}$and $\omega_{d}^{-}$respectively, are expressed as

$$
\begin{gathered}
\omega_{d}^{+}=+\sqrt{2 g k_{0} \cos \phi}+k_{0} v_{0} \cos \theta_{\rho} \\
\omega_{d}^{-}=-\sqrt{2 g k_{0} \cos \phi}+k_{0} v_{0} \cos \theta_{\rho} .
\end{gathered}
$$

Due to the antenna array aperture limitation in the shipborne HFSWR system, the receiving beamwidth $\Delta \theta$ is generally wide so that the Bragg frequency $\omega_{d}$ is a variable within an illuminated clutter patch in the radar footprint by the size of range cell and a beam at a certain azimuth $\theta_{v i}$. Since the Bragg frequency is a function of $\theta_{v}$, the upper and lower Doppler frequency ranges of $\omega_{d}^{+}$and $\omega_{d}^{-}$at a given azimuth $\theta_{v i}$ are calculated by searching the maximum and minimum Bragg frequencies, as given by

$$
\begin{aligned}
& \left\{\min \left[\omega_{d}^{-}\left(\theta_{v i}\right)\right], \max \left[\omega_{d}^{-}\left(\theta_{v i}\right)\right]\right\} \\
& \left\{\min \left[\omega_{d}^{+}\left(\theta_{v i}\right)\right], \max \left[\omega_{d}^{+}\left(\theta_{v i}\right)\right]\right\}
\end{aligned}
$$

where $0 \leqslant \theta_{v}-\frac{\Delta \theta}{2} \leqslant \theta_{v i} \leqslant \theta_{v}+\frac{\Delta \theta}{2} \leqslant \pi$.

At a given scattering ellipse, the eccentricity is defined as the ratio of baseline distance and group range. Provided that we neglect the baseline distance variation due to the ship displacement within the observation time length, the results versus different eccentricities will be the depiction of sea clutter signatures at different group ranges. In Fig. 2, the space-time distribution and spread Doppler ranges corresponding to different eccentricities are illustrated. According to Figs. 2(a) -(b), in comparison with the onshore bistatic HFSWR condition [18], the platform motion deteriorates the symmetry of space-time distribution significantly. As the group range increases, the distribution approaches the monostatic shipborne HFSWR case, where it follows a linear association.

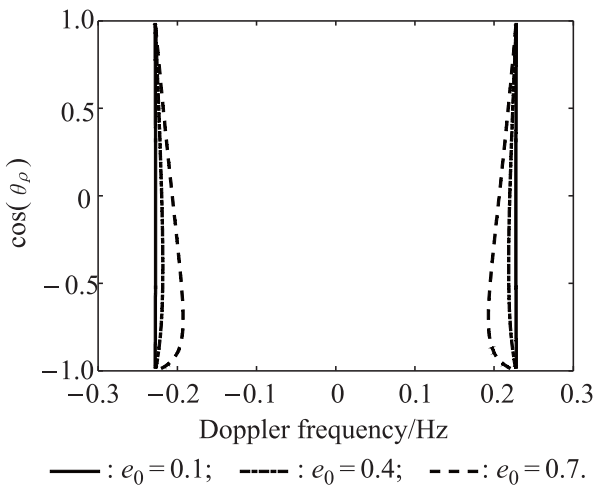

(a) Space-time distribution for the stationary condition

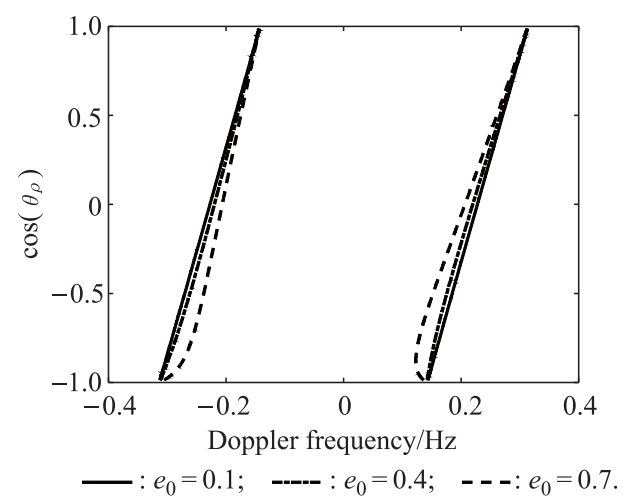

(b) Space-time distribution when the platform is moving

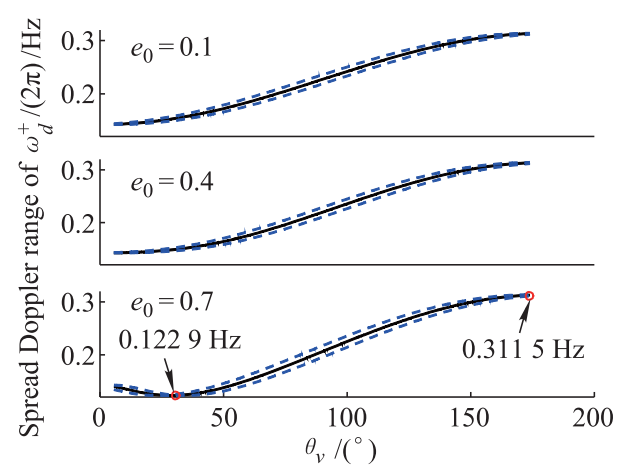

(c) Spread Doppler range of $\omega_{d}^{+}$against $\theta_{v}$

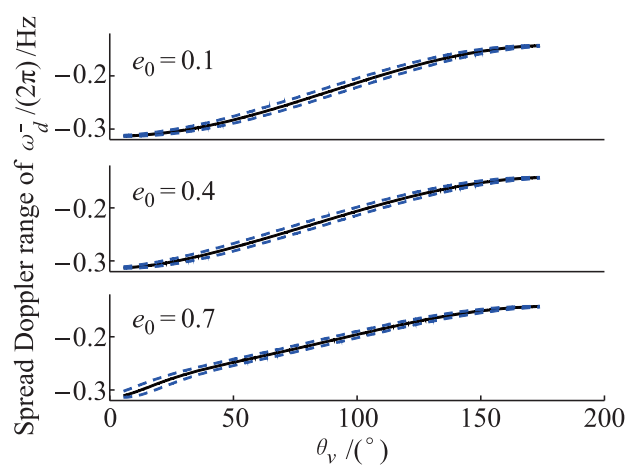

(d) Spread Doppler range of $\omega_{\bar{d}}$ against $\theta_{v}$

Fig. 2 Theoretical space-time distribution and spread Doppler range simulation 
In Figs. 2(c) - (d), the spread Doppler ranges of $\omega_{d}^{+}$and $\omega_{d}^{-}$are simulated against different $\theta_{v}$. The upper and lower of the blue dotted lines indicate the maximum and minimum Bragg frequencies calculated by (20) and (21). And the black solid line is the result directly obtained by (18) and (19) regardless of the effect of receiving beam width. Obviously, when $e_{0}$ is small, the Doppler width discrepancies between the approaching and receding Bragg peaks are insignificant. However, as $e_{0}$ increases, a noticeable difference for the spread range of the approaching Bragg frequency can be seen, compared with other cases. And such discrepancy can manifest its distortions in the cross section calculation results.

\subsection{Simulated cross sections for uniform linear motion}

In this section, simulations are conducted to study the characteristics of the derived cross sections for the uniform linear motion case by lending concreteness with the following four examples.

\subsubsection{Example 1: simulated cross sections for different detection parameters}

In Fig. 3(a), the simulated cross sections are given corresponding to the same assumptions in Fig. 2. To explain the calculation of the Doppler spread range, we take the case of $e_{0}=0.7$ in Fig. 2(c) as an example. As observed, the theoretical spread range is determined by the maximum and minimum Doppler frequencies, which are [0.122 $9 \mathrm{~Hz}, 0.3115 \mathrm{~Hz}$. Thus, the spectral width is $0.1886 \mathrm{~Hz}$. Further, when compared with the simulated result in Fig. 3(a), it is seen that the spread Doppler range is $[0.13 \mathrm{~Hz}, 0.31 \mathrm{~Hz}]$ with the spectral width of $0.18 \mathrm{~Hz}$. The numerical deviation derives from the fact that the Doppler resolution is assumed as $0.01 \mathrm{~Hz}$ when we calculate the cross sections in Fig. 3.

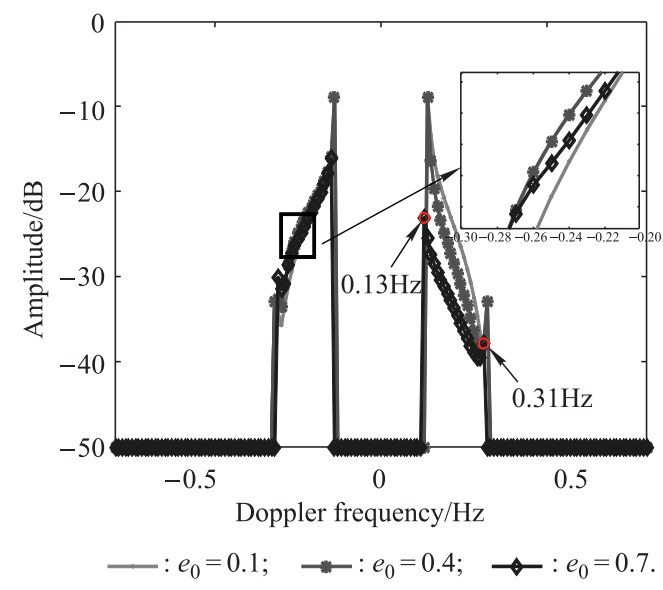

(a) Numerical calculation results against $e_{0}$

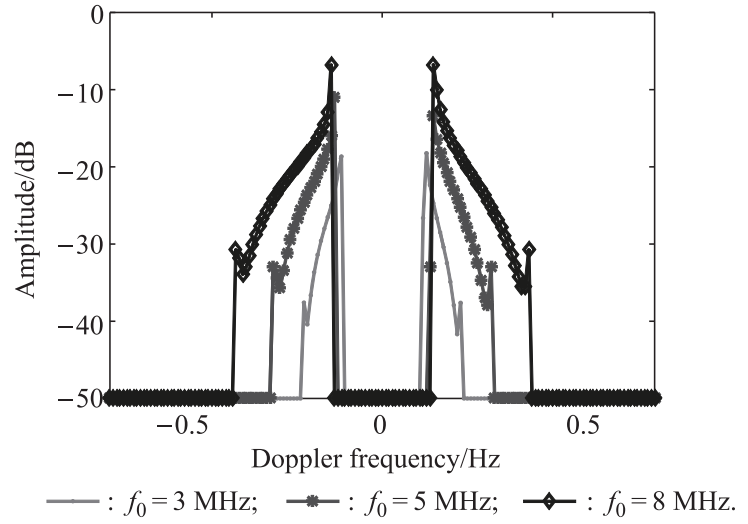

(b) Numerical calculation results against $f_{0}$

Fig. 3 Simulated cross sections for different detection parameters

Regardless of this minor difference, we could conclude that the simulated result agrees well with theoretical calculation result. Similar comparison results can be obtained for other examples in our later simulations. For the sake of simplicity, the compared results are not illustrated.

As $e_{0}$ increases, the negative spectral differences are seen in a minor fluctuation compared with the positive ones. As noted above, this keeps a consistent observation with the space-time distribution discrepancies in Figs. 2(c)-(d). Additionally, Fig. 3(b) illustrates that the increasing frequencies can expand the spectral energy both in magnitude and width. Therefore, as for the shipborne radar system working in a higher operating frequency, it may lead to adverse effects on target detection.

\subsubsection{Example 2: simulated cross sections for different platform motion states}

Fig. 4 gives the numerical calculation results of cross sections versus different $\theta_{0}$ and $v_{0}$. Obviously, the orientation of shipborne platform leads to a rearrangement of spectral energy within the spread Doppler domains. And compared with the stationary situation, the movement of platform spreads the first order sea clutter masking the adjacent Doppler bins around $\pm \sqrt{2 g k_{0} \cos \phi}$ and thus degrades the target discrimination at a low speed.

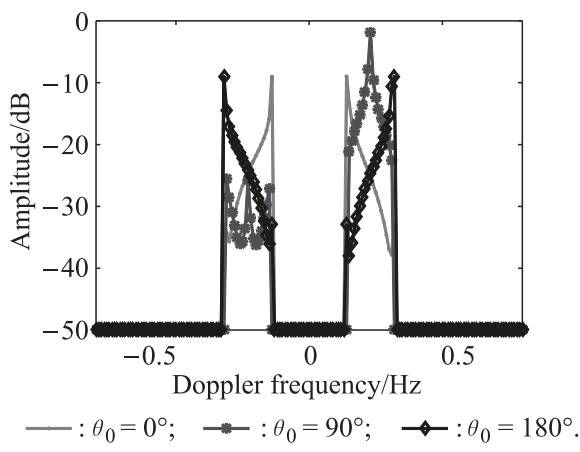

(a) Numerical calculation results against $\theta_{0}$ 


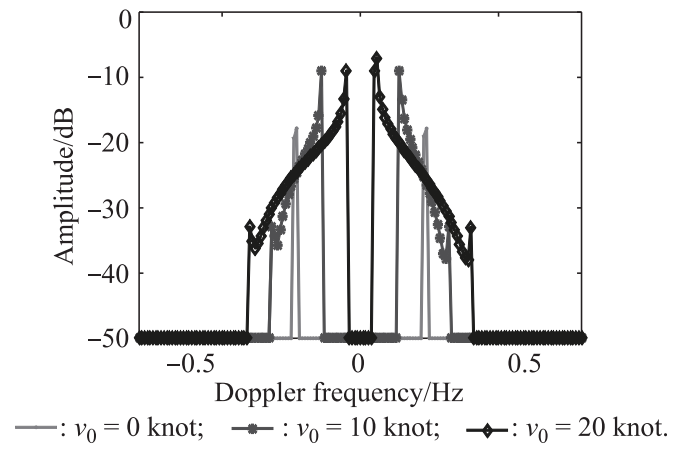

(b) Numerical calculation results against $v_{0}$

Fig. 4 Simulated cross sections for different platform motion states

Along with Fig. 3(b), appropriate considerations should be made between the operating frequencies and motion velocities so as to avoid the possible superposition between the positive and negative spectra. It should be noted that the spectral superposition will be fairly unfavourable for target detection and ocean remote sensing based on the first order sea clutter echoes.

\subsubsection{Example 3: simulated cross sections for different sea environment conditions}

In the following, the results illustrated in Fig. 5 reveal the potential applications in remote sensing areas. Specifically, in Fig. 5(a), the sensitivity of Bragg peak amplitude towards $\theta_{\omega}$ can be used to estimate the wind direction parameter. From [3], this can be achieved by the ratio of Bragg line energies. However, as shown in Fig. 3(a) and Fig. 4(a), the first order sea clutter spectral energy is also influenced by the system parameters and platform motion states. The enhanced spectral complexity is bound to impact on the accuracy and flexibility of sea state parameter measurement in the bistatic shipborne HFSWR system. As for Fig. 5(b), the magnitude of cross sections increases as the wind speed rises and finally achieves saturation for a well-developed ocean surface.

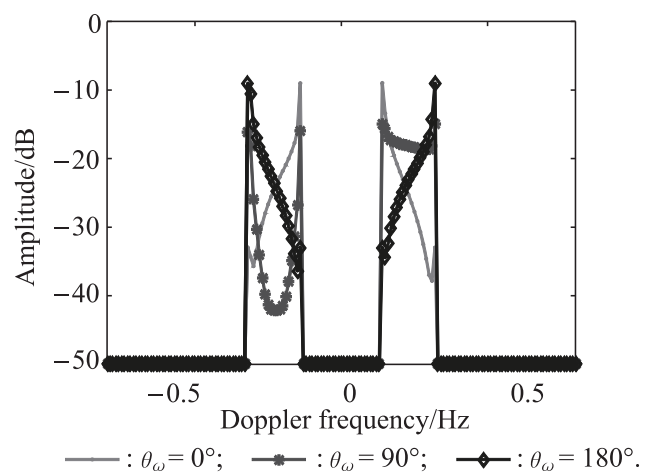

(a) Numerical calculation results against $\theta_{\omega}$

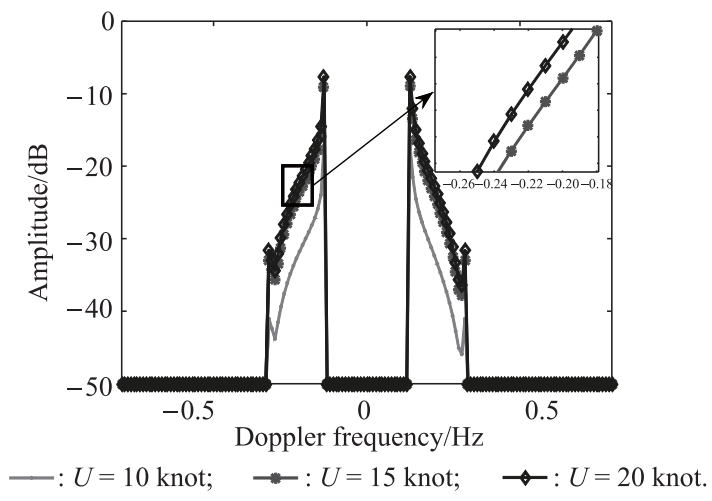

(b) Numerical calculation results against $U$

Fig. 5 Simulated cross sections for different sea environment conditions

\subsubsection{Example 4: results for near range scattering}

The case of near range scattering, i.e. when $e_{0} \rightarrow 1$, is of interest as it dictates totally different sea clutter characteristics compared with the results aforementioned. From Fig. 6(a), at $e_{0}=0.9$, the approaching Bragg peak magnitude is in a reduction of $5 \mathrm{~dB}$ to $20 \mathrm{~dB}$ in contrast with the receding ones. In fact, a reduction of cross section magnitude could severely limit the functionality of HF radar as the sea remote sensor, which depends especially on the location of the noise floor. However, it should be noted that, as the first order sea clutter spectral energy decreases, the small target detection, which is often limited by strong sea clutter echoes, could be improved. In addition, the Doppler occupying width is no longer equivalent. The theoretical spread ranges (the blue dashed line as shown in Fig. 6(a)) calculated by (20) and (21) agree well with the simulated results.

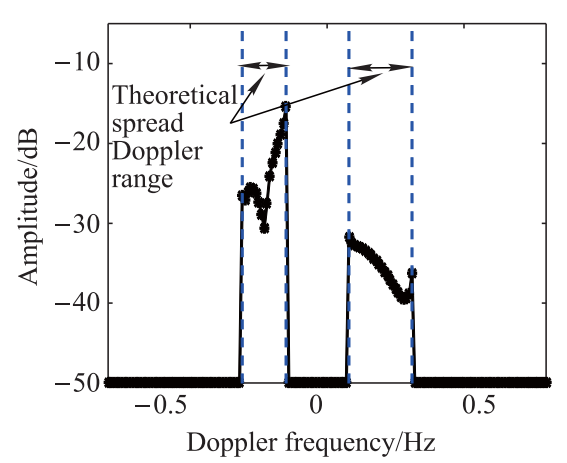

(a) Simulated cross sections

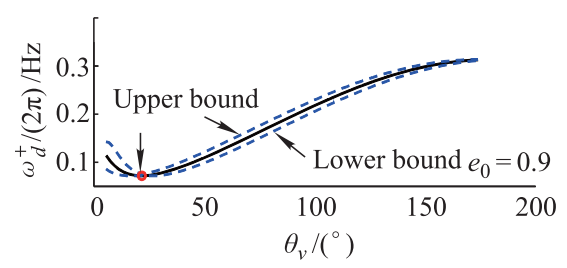




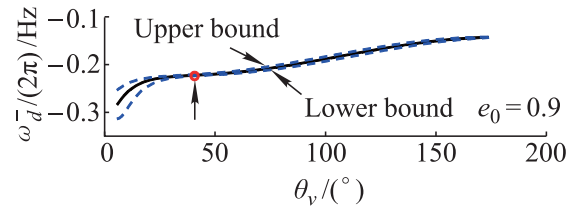

(b) Simulated space-time distribution against $\theta_{v}$

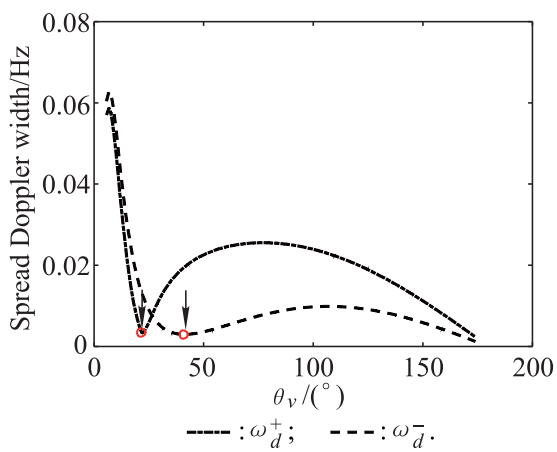

(c) Simulated spread Doppler width against $\theta_{v}$

Fig. 6 Simulated cross sections for near range scattering when $e_{0}=0.9$

In Figs. 6(b) - (c), the space-time distribution and spread Doppler width of $\omega_{d}^{+}$and $\omega_{d}^{-}$at different azimuths are illustrated. The spread Doppler width is the spectral distance between the upper and lower bounds in Fig. 6(b). Therefore, the presence of the valley value in Fig. 6(c) can find its explanation by resorting to the spectral spread ranges marked with red circles in Fig. 6(b). As for each unit clutter patch, the clutter spectral width exhibits apparent spatial differences. Therefore, to ensure a minimum clutter influence both in Doppler width and magnitude for target detection, the obtained results will be useful for setting limitations on the radar coverage area where the target might be more easily detected from the radar data.

\subsection{Simulated cross sections involving the influence of sway motion}

To investigate the impacts on the first order sea clutter cross sections due to the sway motion, three cases are considered whose parameters are summarized in Table 1 [9].

Table 1 Sway motion parameters

\begin{tabular}{ccccc}
\hline Case & $\begin{array}{c}\text { Wind speed } \\
U / \text { knot }\end{array}$ & $\begin{array}{c}\text { Sea } \\
\text { state }\end{array}$ & $\begin{array}{c}\text { Sway } \\
\text { amplitude } \\
a_{s} / \mathrm{m}\end{array}$ & $\begin{array}{c}\text { Sway } \\
\text { frequency } \\
\omega_{s} / 2 \pi / \mathrm{Hz}\end{array}$ \\
\hline Case 1 & 10 & 2 & 0.177 & 0.261 \\
Case 2 & 15 & $3-4$ & 0.581 & 0.174 \\
Case 3 & 20 & 5 & 1.228 & 0.127 \\
\hline
\end{tabular}

By invoking (16), numerical calculation results corresponding to Table 1 are illustrated in Fig. 7. It is straightforward to notice that the sway motion will cause the first order sea clutter spectral energy spreading into the near-in Doppler bins, which can be understood as the Doppler and amplitude modulation of the results of uniform linear motion case. Such influence can find its explanation through the Bessel function embodied in (16). Evidently, the modulated clutter energy is proportional to the sway magnitude as well as the wind speed and finally achieves saturation at a high sea state. In this case, the second order spectra will be contaminated by the motion-induced spread clutter contents rendering them useless for wind and wave measurements. Thus, some compensation methods are needed to eliminate the influence imposed by the sway motion.

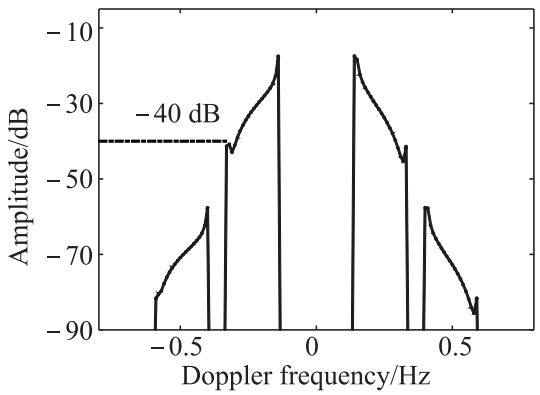

(a) Case 1

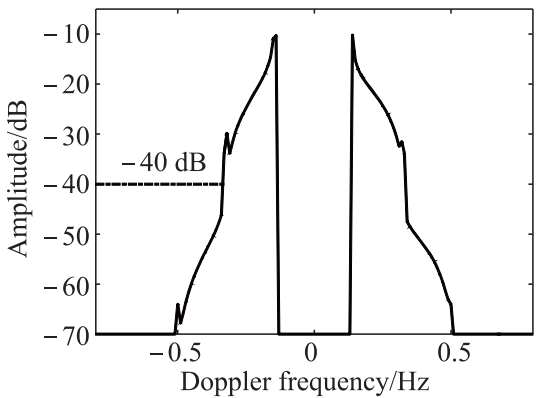

(b) Case 2

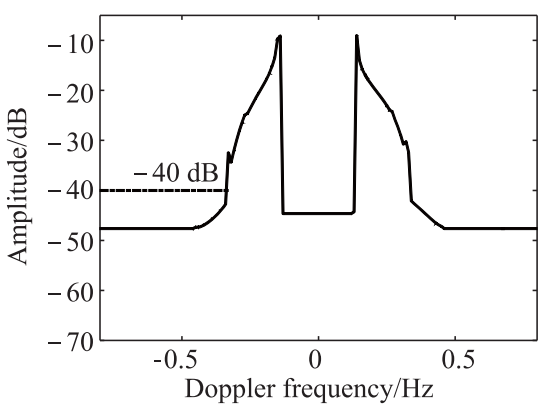

(c) Case 3

Fig. 7 Effects on the first order sea clutter cross sections due to the sway motion

\section{Conclusions}

In this paper, we present the results of a simulation study of the first order sea clutter Doppler spectra for bistatic shipborne HFSWR. This has been achieved by deriving a closed-form of cross sections, which takes account of the bistatic deployment and two specified ship motion conditions. According to the simulation results, the forward motion of the shipborne platform results in the sea clutter 
Doppler spectra spreading severely. The extent of spectral spreading is largely restricted by the operating parameters, platform moving speed, as well as the detection range. The Doppler modulation arising from the sway motion component has no prominent contributions to the spectral width and amplitude, which can be regarded as a modulation of the results from uniform linear motion. The extra modulated spectral energy will be mixed with the second or higher order sea clutter components and this may bring negative influence on applications in remote sensing significantly.

In general, the clutter magnitude and width are seen to exhibit significant discrepancies against different platform motion states and ocean environment conditions. The obtained result is a crucial observation in setting appropriate limits on the system operating parameters when using such a setup for target detection or ocean wave measurement.

\section{References}

[1] Y. Liu. Target detection and tracking with a high frequency ground wave over-the-horizon radar. Proc. of the CIE International Conference on Radar, 1996: 29-33.

[2] J. Xie, Y. Yuan, Y. Liu. Experimental analysis of sea clutter in shipborne HFSWR. IEE Radar, Sonar and Navigation, 2001, 148(2): $67-71$.

[3] B. J. Lipa, D. E. Barrick. Extraction of sea state from HF radar sea echo: mathematical theory and modeling. Radio Science, 1986, 21(1): $81-100$.

[4] S. Grosdidier, A. Baussard, A. Khenchaf. HFSW radar model: simulation and measurement. IEEE Trans. on Geoscience and Remote Sensing, 2010, 48(9): 3539-3549.

[5] S. Grosdidier, P. Forget, Y. Barbin, et al. HF bistatic ocean Doppler spectra: simulation versus experimentation. IEEE Trans. on Geoscience and Remote Sensing, 2014, 52(4): $2138-2148$.

[6] E. W. Gill, J. Walsh. High-frequency bistatic cross sections of the ocean surface. Radio Science, 2001, 36(6): 1459-1475.

[7] B. J. Lipa, D. E. Barrick, J. Isaacson, et al. CODAR wave measurements from a north sea semisubmersible. IEEE Journal of Oceanic Engineering, 1990, 15(2): 119-125.

[8] M. Lesturgie. Use of STAP techniques to enhance the detection of slow targets in shipborne HFSWR. Proc. of the International Radar Conference, 2003: 504-509.

[9] J. Walsh, W. Huang, E. W. Gill. The first-order high frequency radar ocean surface cross section for an antenna on a floating platform. IEEE Trans. on Antennas and Propagation, 2010, 58(9): $2994-3003$.

[10] J. Walsh, W. Huang, E. W. Gill. The second-order high frequency radar ocean surface cross section for an antenna on a floating platform. IEEE Trans. on Antennas and Propagation, 2012, 60(10): $4804-4813$.

[11] J. E. Khoury, R. Guinvarc'h, R. Gillard, et al. Sea-echo doppler spectrum perturbation of the received signals from a floating high-frequency surface wave radar. IET Radar, Sonar and $\mathrm{Na}$ - vigation, 2012, 60(3): $165-171$.

[12] J. Xie, M. Sun, Z. Ji, et al. First-order ocean surface crosssection for shipborne HFSWR. Electronics Letters, 2013, 49(16): $1025-1026$.

[13] M. Sun, J. Xie, Z. Ji, et al. Second-order ocean surface cross section for shipborne HFSWR. IEEE Antennas and Wireless Propagation Letters, 2014, 14: 823-826.

[14] H. Sun, X. Guo, Y. Lu, et al. Estimation of the ocean clutter rank for HF/UHF radar space-time adaptive processing. IET Radar, Sonar and Navigation, 2010, 4(6): $755-763$.

[15] J. Xie, Z. Wang, Z. Ji, et al. High-resolution ocean clutter spectrum estimation for shipborne HFSWR using sparserepresentation-based MUSIC. IEEE Journal of Oceanic Engineering, 2014, 40(3): 546-557.

[16] J. Walsh, E. W. Gill. An analysis of the scattering of highfrequency electromagnetic radiation from rough surfaces with application to pulse radar operating in backscatter mode. $R a$ dio Science, 2000, 35(6): 1337 - 1359.

[17] C. Liu, B. Chen, D. Chen, et al. Analysis of first-order sea clutter in a shipborne bistatic high frequency surface wave radar. Proc. of the CIE International Conference on Radar, 2006: $1-4$.

[18] J. Xie, M. Sun, Z. Ji. Space-time model of the first-order sea clutter in onshore bistatic high frequency surface wave radar. IET Radar, Sonar and Navigation, 2015, 9(1): 55-61.

\section{Biographies}

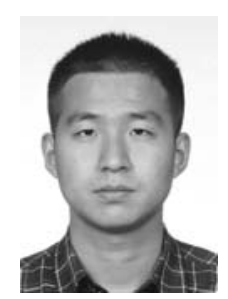

Yongpeng Zhu was born in 1988. He received his B.S. and M.S. degrees from Harbin Institute of Technology (HIT) in 2011 and 2014, respectively. $\mathrm{He}$ is currently working toward his Ph.D. degree in information and communication engineering from HIT. His research interests include fields of signal processing and radar system simulation in $\mathrm{HF}$ OTH radar.

E-mail: zhuyp@hit.edu.cn

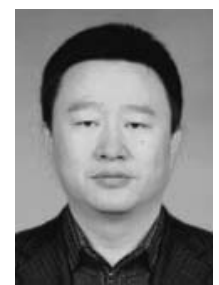

Yinsheng Wei was born in 1974. He received his M.S. and Ph.D. degrees in communication and information systems from HIT in 1998 and 2002, respectively. He joined the Department of Electronics Engineering in HIT as a lecturer, and became a professor in 2011. He is a member of IEEE AES, and a senior member of CIE. His main research interests include radar signal processing and radar system analysis and simulation.

E-mail: weiys@ hit.edu.cn

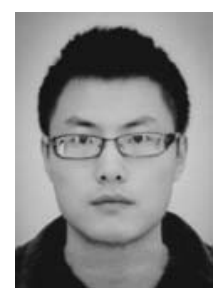

Peng Tong was born in 1987. He received his M.S. degree in information and communication engineering from HIT, in 2012. He is currently working toward his Ph.D. degree at HIT. His current research interest is adaptive signal processing in high frequency radar.

E-mail: tongpeng@hit.edu.cn 